\title{
Early mortality in patients with community- acquired pneumonia: causes and risk factors
}

\author{
C. Garcia-Vidal*, N. Fernández-Sabé*, J. Carratalà*, V. Díaz*, R. Verdaguer", \\ J. Dorca", F. Manresa" and F. Gudiol*
}

ABSTRACT: The first $48 \mathrm{~h}$ of evolution of patients with community-acquired pneumonia (CAP) are critical. The aim of the present study was to determine the frequency, causes and factors associated with early mortality in CAP.

Nonimmunocompromised adults hospitalised with CAP were prospectively observed from 1995 to 2005. Early deaths, defined as death due to any cause $\leqslant \mathbf{4 8} \mathrm{h}$ after admission, were compared with all patients who survived $>48 \mathrm{~h}$. Furthermore, early deaths were compared with late deaths (patients who died $>48 \mathrm{~h}$ ) and with survivors.

Of 2,457 patients, $57(2.3 \%)$ died $\leqslant 48 \mathrm{~h}$ after admission. Overall mortality was $7.7 \%$. The main causes of early mortality were respiratory failure and septic shock/multiorgan failure. Independent factors associated with early deaths were increased age, altered mental status at presentation, multilobar pneumonia, shock at admission, pneumococcal bacteraemia and discordant empiric antibiotic therapy.

Currently, early mortality is relatively low and is caused by pneumonia-related factors. It occurs mainly among the elderly and in patients presenting with altered mental status, multilobar pneumonia and septic shock. Pneumococcal bacteraemia and discordant antibiotic therapy, mainly due to lack of coverage against Pseudomonas aeruginosa are also significant risk factors.

KEYWORDS: Community-acquired pneumonia, early mortality, Pseudomonas aeruginosa, shock, Streptococcus pneumoniae

ommunity-acquired pneumonia (CAP) continues to be a major health problem worldwide [1-3]. Despite more accurate aetiological diagnosis, effective antibiotic therapy and advances in supportive care, the morbidity and mortality rates associated with this infection remain high. Recent studies report complications in $15-50 \%$ of hospitalised patients and overall mortality rates that range from $\sim 10 \%$ for patients treated in a hospital setting to $>30 \%$ for patients treated in an intensive care unit [4-7]. According to these studies, most deaths occurring within 30 days of presentation appear to be pneumonia related. A substantial number of identifiable risk factors may influence mortality, and some of the factors associated with mortality within the first days may differ from those associated with mortality occurring later.

In this setting, information regarding the causes and factors related to mortality within the first $48 \mathrm{~h}$ of pneumonia are scarce and there is no clear consensus on whether this very early mortality is modifiable by medical intervention [8-10].

The aim of the present study was to determine the frequency, causes and factors associated with early mortality in a large prospective cohort of hospitalised patients with CAP.

\section{MATERIALS AND METHODS}

\section{Study subjects and design}

The study was carried out in a 900-bed university hospital for adults in Barcelona, Spain. The hospital serves an area with 1,100,000 inhabitants and admits $\sim 24,000$ patients per year. All nonimmunocompromised patients with CAP who were admitted to the hospital from February 1995 to December 2005 were prospectively recruited and followed up. Patients with neutropenia or HIV infection and those who had undergone transplantation were not included. For the purposes of the present study, patients were divided into two groups: those who died due to any cause $\leqslant 48 \mathrm{~h}$ after admission (early
AFFILIATIONS

*Infectious Disease,

${ }^{*}$ Microbiology, and

"Respiratory Medicine Services,

Institute of Biomedical Investigation of Bellvitge (IDIBELL) - Hospital University of Bellvitge, University of Barcelona, L'Hospitalet, Barcelona, Spain.

CORRESPONDENCE

C. Garcia-Vidal

Infectious Disease Service Hospital University of Bellvitge Feixa Llarga s/n 08907 L'Hospitalet Barcelona

Spain

Fax: 34932607637

E-mail: carolgv75@hotmail.com

Received:

September 282007

Accepted after revision:

May 012008

\section{SUPPORT STATEMENT}

This study was supported by research grant REIPI RD06/0008 from the

Ministry of Health and Consumption, Institute of Health Carlos III, Spanish Network for the Research in Infectious Diseases; and by the Institute of Biomedical Investigation of Bellvitge (C. Garcia-Vidal). The funding sources had no role: in the study design; in the collection, analysis or interpretation of the data; or in the decision to submit the manuscript for publication. Only the authors had full access to the data files for the study.

STATEMENT OF INTEREST

None declared.

European Respiratory Journal Print ISSN 0903-1936 Online ISSN 1399-3003 
deaths), and those who survived the first $48 \mathrm{~h}$ after admission. In addition, the latter group was divided into two: patients who died $>48 \mathrm{~h}$ after admission (late deaths) and survivors. This prospective, longitudinal and observational study was approved by the Hospital University of Bellvitge (Barcelona, Spain) Ethical Committee.

\section{Clinical evaluation and follow-up}

At the initial visit, and before starting empirical antibiotic therapy, patients underwent a complete clinical history and physical examination. Basic chemistry and haematology tests, arterial blood gas determinations and chest radiography were performed. Two sets of blood samples were obtained and cultured and, when available, a sputum sample was evaluated by use of Gram staining and culture. Invasive procedures and urinary antigen detection for Streptococcus pneumoniae and Legionella pneumophila were performed if indicated by the attending physician. Paired serum samples obtained during the acute and convalescent phases of infection (separated by a 3-8 week interval) were also obtained for serological studies.

Patients were seen daily during their hospital stay by one or more of the investigators, who provided medical advice when requested and recorded: demographic characteristics; underlying disease; clinical features; vaccination status; causative agents; therapy; and outcomes in a computer-assisted protocol.

\section{Definitions}

CAP was defined as the presence of a new infiltrate on chest radiography plus at least one of the following: fever (temperature $\geqslant 38.0^{\circ} \mathrm{C}$ ) or hypothermia (temperature $\leqslant 35.0^{\circ} \mathrm{C}$ ); new cough, with or without sputum production; pleuritic chest pain; dyspnoea; or altered breath sounds on auscultation. The diagnosis of septic shock was based on a systolic blood pressure of $<90 \mathrm{mmHg}$ and peripheral hypoperfusion with clinical or bacteriological evidence of uncontrolled infection. Complications were defined as any untoward circumstance occurring during hospitalisation, with the exception of sideeffects from medication. Antibiotic therapy was administered according to the hospital guidelines, which recommended the administration of a $\beta$-lactam (ceftriaxone or amoxicillin clavulanate) with or without a macrolide (erythromycin or clarithromycin) or a fluoroquinolone. Combination therapy was recommended for patients with clinical suspicion of Legionella or an atypical pathogen, or in the absence of a demonstrative sputum Gram stain. From February 2000 onwards, levofloxacin monotherapy was permitted in selected cases. Concordance of antibiotic therapy was examined for all cases with an aetiological diagnosis according to susceptibility test criteria for classic respiratory pathogens. Early death was defined as death due to any cause $\leqslant 48 \mathrm{~h}$ after hospitalisation; late death was defined as a death due to any cause $>48 \mathrm{~h}$ $<30$ days after hospitalisation. Overall, mortality was defined as death due to any cause $<30$ days after hospitalisation. The severity of illness at presentation was quantified using the validated Pneumonia Outcome Research Team prediction rule for 30-day mortality and medical complications in CAP [4].

\section{Microbiological studies}

The aetiological diagnosis of CAP was established as described elsewhere [11]. Isolation of Legionella was attempted in sputum and other respiratory samples by using selective media (buffered charcoal-yeast extract medium- $\alpha$ ). Detection of L. pneumophila serogroup I antigen in urine was performed by an immunoenzymatic commercial method (Legionella Urinary Antigen; Binax, Portland, ME, USA). Detection of the $S$. pneumoniae antigen in urine was performed by a rapid immunochromatographic assay (Now ${ }^{\mathrm{TM}}$; Binax). Standard serological methods were used to determine antibodies against the following pathogens: Mycoplasma pneumoniae (indirect agglutination), Chlamydia psittaci (immunofluorescence (IF)), Chlamydia pneumoniae (micro-IF), Coxiella burnetii (IF), and L. pneumophila (serogroups 1-6; enzyme immunoassay (EIA)). The Centers for Disease Control and Prevention criteria [12] were used for Chlamydia pneumoniae (micro-IF) serology. Serologies for respiratory syncytial virus (EIA), parainfluenza 3 virus (EIA) and influenza A virus (EIA) were performed as part of a research protocol during the first years of the study.

The antibiotic sensitivity of all isolates was determined at the Laboratory of the Microbiology Service, Bellvitge University Hospital (Barcelona, Spain), by using a commercial microdilution panel (STRHAEl, Sensititre; Trek Diagnostic Systems Ltd, East Grinstead, England) in accordance with the National Committee for Clinical Laboratory Standards guidelines [13]. The National Committee for Clinical Laboratory Standards 2001 criteria were used to define susceptibility of pneumococcal isolates [14].

\section{Analysis}

In order to assess factors associated with early mortality, early deaths were compared with the remaining patients. Early deaths were compared with late deaths in order to more accurately discern risk factors for early mortality and late mortality. To detect significant differences between groups the following were used: Chi-squared test with continuity correction for categorical variables and paired t-test for continuous variables. All the significant variables detected in the univariate analysis and considered clinically relevant were involved in the multivariate analysis. The analysis was performed with a stepwise logistic-regression model. In all analyses, $\mathrm{p}<0.05$ was considered to be statistically significant. All reported p-values are two tailed.

\section{RESULTS}

A total of 2,457 hospitalised patients with CAP were included in the present study. Out of these, $57(2.3 \%)$ were early deaths $(\leqslant 48 \mathrm{~h}), 131(5.4 \%)$ were late deaths and 2,269 (92.3\%) were survivors. Overall mortality (<30 days) was 7.7\% (188 patients). Demographic characteristics and the main clinical features of patient groups are compared in table 1. Early deaths were older and more often classified as having a highrisk pneumonia, compared with the remaining patients. Altered mental status, renal failure, tachycardia, increased respiratory rate, high fever, multilobar infiltrates, respiratory failure and shock were more frequently found at baseline in early deaths. Late deaths had greater comorbidity than early deaths, mainly chronic heart disease and chronic renal disease. Conversely, shock at admission and bacteraemic pneumonia was more frequent in early deaths. 
TABLE 1 Main demographic and clinical characteristics of 2,457 patients hospitalised for community-acquired pneumonia

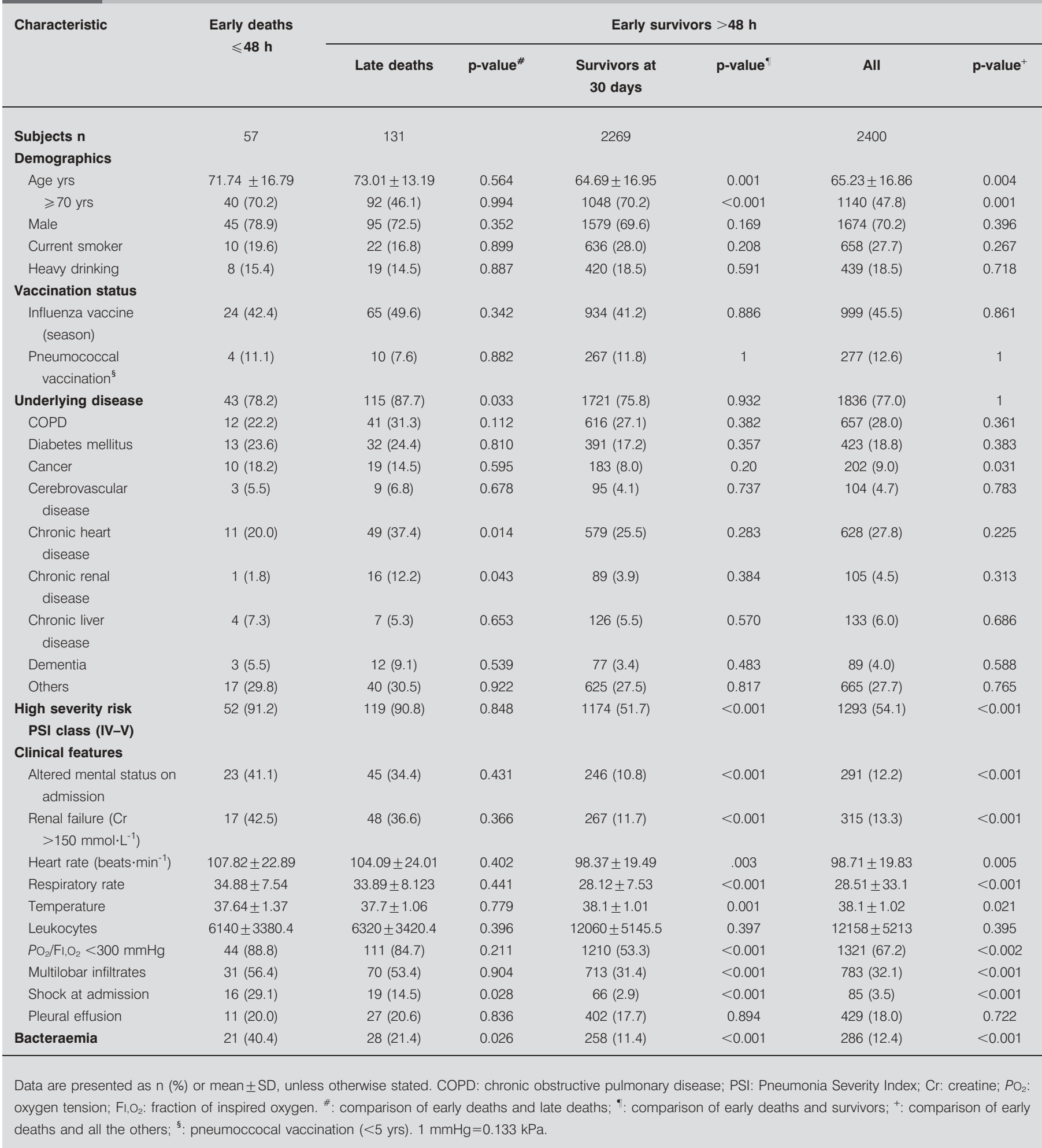

Causes of early mortality are shown in table 2. Acute respiratory failure secondary to pneumonia and multiorgan failure associated with septic shock were the most frequent.

As shown in table 3, S. pneumoniae was the most frequently identified pathogen; there were no differences between groups.
Bacteraemic pneumococcal pneumonia was significantly more frequent in early and late deaths. Out of 324 S. pneumoniae strains isolated from the 718 patients with pneumococcal pneumonia, no significant differences were found in the rates of antibiotic resistance between groups (early deaths versus late deaths versus survivors versus all (late deaths and survivors)): 


\begin{tabular}{lcc}
\hline TABLE 2 & $\begin{array}{l}\text { Causes of early and late death in patients } \\
\text { hospitalised with community-acquired } \\
\text { pneumonia }\end{array}$ & Late deaths \\
\hline Cause of death & Early deaths & 131 \\
\hline $\begin{array}{l}\text { Subjects } \\
\text { Acute respiratory failure }\end{array}$ & $38(66.6)$ & $64(48.8)$ \\
$\begin{array}{l}\text { Septic shock / multiorgan } \\
\text { failure }\end{array}$ & $14(24.6)$ & $22(16.8)$ \\
$\begin{array}{l}\text { Congestive heart failure } \\
\text { or cardiac arrhythmia }\end{array}$ & $4(7.0)$ & $16(12.2)$ \\
$\begin{array}{l}\text { Diabetic ketoacidosis } \\
\text { Nosocomial infection } \\
\text { Others }\end{array}$ & $1(1.7)$ & $0(0)$ \\
\end{tabular}

Data are presented as $n$ or $n(\%)$.

penicillin (Minimum Inhibitory Concentration (MIC) $\geqslant 4 \mu \mathrm{g} \cdot \mathrm{mL}^{-1} ; \quad 20.0 \%$ versus $8.7 \% \quad(\mathrm{p}=0.738)$, versus $8.1 \%$ $(p=0.448)$, and versus $8.3 \%(p=0.521))$, cefotaxime/ceftriaxone (MIC $\geqslant 2 \mu \mathrm{g} \cdot \mathrm{mL}^{-1} ; 0 \%$ versus $0 \%(\mathrm{p}=1)$, versus $6.3 \%(\mathrm{p}=0.563)$, and versus $6.4 \%(\mathrm{p}=0.586)$, erythromycin (MIC $\geqslant 1 \mu \mathrm{g} \cdot \mathrm{mL}^{-1}$; $37.5 \%$ versus $15.8 \%(p=0.464)$, versus $13.7 \%(p=0.058)$, and versus $13.4(\mathrm{p}=0.088)$ ), and ciprofloxacin (MIC $\geqslant 4 \mu \mathrm{g} \cdot \mathrm{mL}^{-1} ; 0 \%$ versus $0 \%(p=1)$, versus $1.9 \%(p=1)$, and versus $1.75 \%(p=1))$. Pneumonia due to Gram-negative bacilli was significantly more frequent in patients who died (early and late deaths), especially in the case of Pseudomonas aeruginosa pneumonia. No significant differences were observed regarding the frequency of $P$. aeruginosa between nursing home residents and the remaining patients ( 1.9 versus $0.8 \% ; \mathrm{p}=0.361$ ).

As shown in table 4, most patients were initially treated with a single antimicrobial agent. Concordance of antibiotic therapy could be determined in 38 out of 57 early deaths, 85 out of 131 late deaths, and 1,223 out of 2,269 survivors. Overall, early deaths received discordant antibiotic therapy more frequently than all others. Discordant empirical antibiotic therapy in early deaths was mainly due to lack of coverage against $P$. aeruginosa infection (five out of six patients; 83\%). All these five patients were aged $>70 \mathrm{yrs}$ and were classified into the Pneumonia Severity Index (PSI) risk class V; three of them had bacteraemia and septic shock and two had chronic obstructive pulmonary disease (COPD), but none had bronchiectasis or were receiving chronic steroid therapy.

Table 5 shows factors associated with early mortality by multivariate analyses. After adjustment, factors associated with early death $(\leqslant 48 \mathrm{~h})$ were increased age, altered mental status, multilobar pneumonia, shock at admission, pneumococcal bacteraemia and discordant empiric antibiotic therapy. No significant differences were found in these factors associated with death when analysing 5-day instead of 2-day mortality, by univariate and multivariate analyses. Among all 188 patients who died, shock at admission was independently associated with early deaths (odds ratio (OR) 2.683, 95\% confidence interval (CI) 1.014-7.097), whereas chronic heart disease was associated with late deaths (OR 0.382, 95\% CI $0.153-0.956)$.

TABLE 3 Aetiology of community-acquired pneumonia of 2,457 patients hospitalised for community-acquired pneumonia

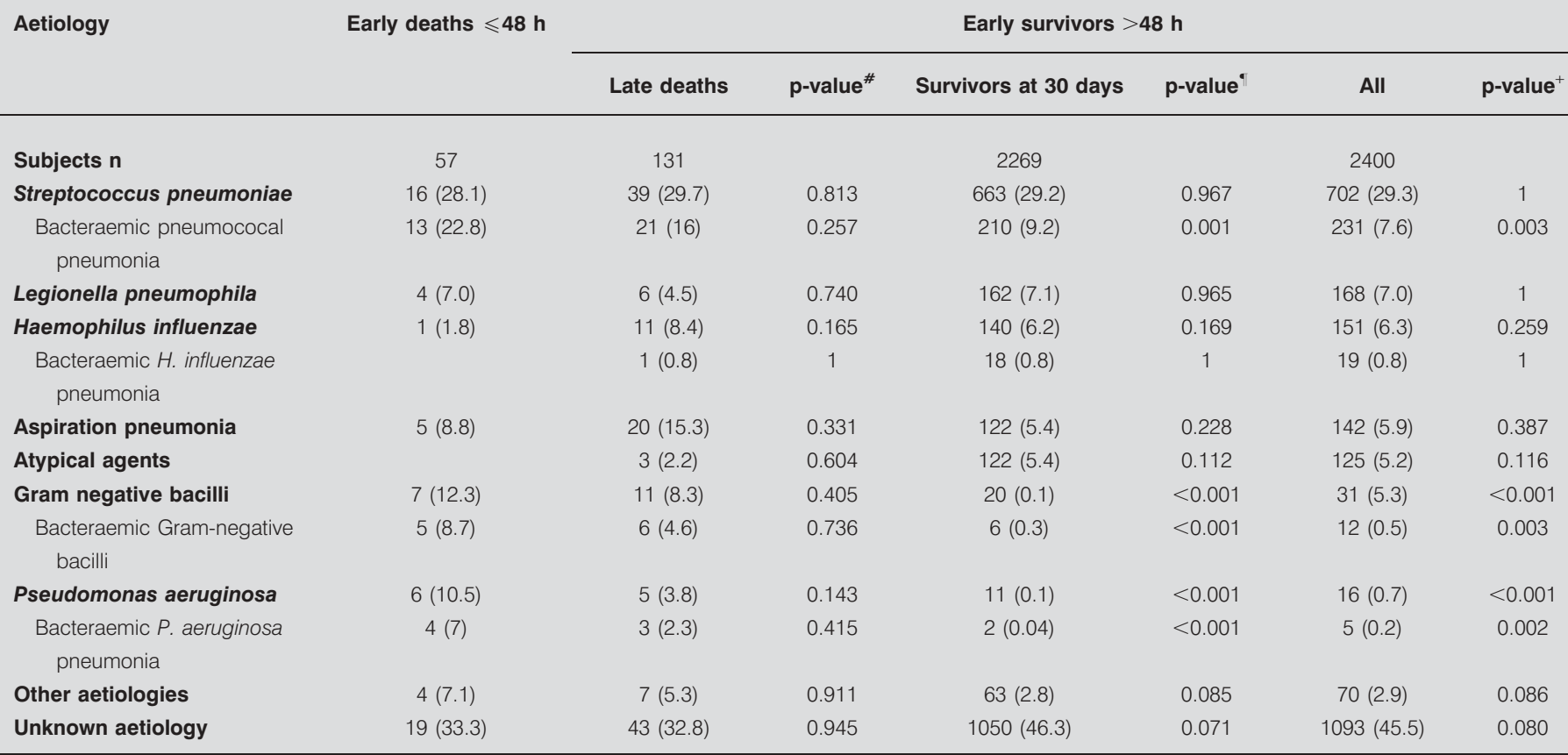

Data are presented as $\mathrm{n}(\%)$, unless otherwise stated. ${ }^{*}$ : comparison of early mortality and late deaths; " : comparison of early deaths and survivors. ${ }^{+}$: comparison of early deaths and all the others. 
TABLE 4 Antibiotic therapy of 2,457 patients hospitalised for community-acquired pneumonia

\begin{tabular}{|c|c|c|c|c|c|c|c|}
\hline Therapy & $\begin{array}{l}\text { Early deaths } \\
\quad \leqslant 48 \mathrm{~h}\end{array}$ & Late deaths & p-value ${ }^{\#}$ & $\begin{array}{l}\text { Survivors at } \\
30 \text { days }\end{array}$ & p-value & All & p-value ${ }^{+}$ \\
\hline Subjects & 57 & 13 & & 2269 & & 2400 & \\
\hline \multicolumn{8}{|l|}{ Initial antibiotic therapy } \\
\hline Macrolides & 2 & 3 & 0.987 & 159 & 0.766 & 162 & 0.379 \\
\hline Quinolones & & 6 & 0.233 & 255 & 0.007 & 261 & 0.004 \\
\hline Other & 2 & 2 & 0.752 & 57 & 0.235 & 59 & 0.735 \\
\hline Combination therapy & $21(36.8)$ & $40(30.5)$ & 0.402 & $514(22.7)$ & 0.018 & $554(23.1)$ & 0.025 \\
\hline$\beta$-lactams + macrolides & 12 & 20 & 0.331 & 197 & 0.093 & 201 & 0.003 \\
\hline$\beta$-lactams + quinolones & 7 & 19 & 0.860 & 278 & 0.099 & 297 & .146 \\
\hline Monotherapy & $33(63)$ & $89(74.8)$ & 0.4 .83 & $862(73.4)$ & 0.146 & 951 (73.5) & 0.113 \\
\hline$\beta$-Lactams & 31 & 76 & 0.735 & 735 & 0.210 & 811 & 0.124 \\
\hline Macrolides & & 3 & 0.553 & 41 & 0.395 & 44 & 0.394 \\
\hline Quinolones & & 9 & 0.312 & 60 & 0.160 & 69 & 0.086 \\
\hline Other & 2 & 1 & 0.177 & 26 & 0.276 & 27 & 0.583 \\
\hline Combination therapy & 19 (36.5) & $30(25.2)$ & 0.483 & $312(26.6)$ & 0.146 & $342(26.5)$ & 0.113 \\
\hline$\beta$-lactams + macrolides & 11 & 14 & 0.636 & 110 & 0.046 & 124 & 0.010 \\
\hline$\beta$-lactams + quinolones & 7 & 16 & 0.404 & 190 & 0.396 & 206 & 0.846 \\
\hline Other combinations & 1 & 0 & 0.400 & 12 & 0.815 & 12 & 0.815 \\
\hline $\begin{array}{l}\text { Initial antibiotic therapy in } \\
\text { pneumoccocal pneumonia }\end{array}$ & 16 & 39 & & 663 & & 702 & \\
\hline Combination therapy & $4(25)$ & $13(33.3)$ & 0.749 & $140(21.1)$ & 0.757 & $153(21.8)$ & 0.763 \\
\hline
\end{tabular}

\section{DISCUSSION}

It is widely recognised that the evolution of patients with CAP within the first $48 \mathrm{~h}$ is crucial $[1,15,16]$. In fact, once clinical stability is achieved, substantial clinical deterioration owing to pneumonia is rare [17]. In a previous study, the current authors analysed the causes and factors associated with early failure in hospitalised patients with CAP [11]. In the definition of early failure as "lack of response or worsening of clinical or radiological status at $48-72 \mathrm{~h}$ requiring changes in antibiotic therapy or invasive procedures," patients who had died within the first $48 \mathrm{~h}$ of admission were specifically excluded. The current prospective study offers a comprehensive evaluation of this group of patients in order to establish the causes of, and risk factors for, early mortality in CAP.

In the present series of patients admitted according to predefined criteria [18], in which the severe immunosuppressed population is excluded, the early mortality rate was $2.3 \%$; that is, one third of the total patients with CAP who died during hospital admission. This figure, though relatively low in the current authors' view, is difficult to compare with others obtained in previous series, owing to differences in definitions and study populations.

Overall, although differences in the frequency and types of underlying diseases were not observed among early deaths compared with all the others, the demographic and clinical characteristics of early deaths define a group with more severe pneumonia, as shown by the fact that $91 \%$ of cases were classified in the PSI high severity risk classes.

The most frequent causes of early death were respiratory failure and shock/multiorgan failure. Indeed, a large proportion of them had bacteraemia and presented with septic shock and/or respiratory failure at admission. The vast majority of deaths were pneumonia related, in the setting of an unbalanced inflammatory response. 


\begin{tabular}{|c|c|c|c|}
\hline \multirow{2}{*}{$\begin{array}{l}\text { TABLE } 5 \\
\text { Risk factor }\end{array}$} & \multicolumn{3}{|c|}{$\begin{array}{l}\text { Risk factors associated with early deaths in } 2,457 \\
\text { patients hospitalised with community-acquired } \\
\text { pneumonia by multivariate analysis }\end{array}$} \\
\hline & & OR & $95 \% \mathrm{Cl}$ \\
\hline \multicolumn{2}{|l|}{ Male sex } & 0.538 & $0.254-1.140$ \\
\hline \multicolumn{2}{|l|}{ Age $\geqslant 70$ yrs } & $2.727^{\circ}$ & $1.394-5.337$ \\
\hline \multicolumn{2}{|c|}{$\begin{array}{l}\text { Altered mental status at } \\
\text { admission }\end{array}$} & $2.481^{\circ}$ & $1.276-4.822$ \\
\hline \multicolumn{2}{|c|}{ Shock at admission } & $7.547^{\circ}$ & 3.453-16.494 \\
\hline \multicolumn{2}{|c|}{ Respiratory failure ${ }^{\#}$} & 2.073 & $0.848-5.067$ \\
\hline \multicolumn{2}{|c|}{ Multilobar pneumonia } & $1.979^{\circ}$ & $1.042-3.758$ \\
\hline \multicolumn{2}{|c|}{ Discordant antibiotic therapy } & $11.281^{\circ}$ & 3.497-36.387 \\
\hline \multicolumn{2}{|c|}{$\begin{array}{l}\text { Bacteraemic pneumococcal } \\
\text { pneumonia }\end{array}$} & $2.373^{\circ}$ & $1.083-5.200$ \\
\hline
\end{tabular}

Both bacteraemic pneumococcal pneumonia and P. aeruginosa pneumonia were significantly more frequent in early deaths. In the case of $S$. pneumoniae, no relationship between mortality (early and late deaths) and drug resistance could be demonstrated. This observation is in agreement with most previous studies of bacteraemic pneumococcal pneumonia which have not shown differences in mortality between those with susceptible and those with nonsusceptible pneumococci when controlling for age, underlying disease, severity of illness on presentation, and appropriate treatment $[19,20]$. In fact, all patients who died early and had pneumococcal infection were given concordant antibiotic therapy from admission. This evidence reinforces the classical concept that early deaths are less dependent on antibiotic efficacy than on other factors, including inadequate host response [21]. Recent studies suggest that modulation of the immune system could improve the outcomes of patients with severe pneumonia [22, 23]. However, further studies are warranted to evaluate the relationship among excessive host response and early deaths. Importantly, randomised clinical trials addressing the potential role of steroids as adjunctive therapy in severe CAP are needed.

Conversely, it has been shown previously that polysaccharide pneumococcal vaccination may prevent invasive pneumococcal disease in adults, and improve outcomes [24, 25]. In the present study, $<10 \%$ of patients who died had received pneumococcal vaccination, despite the fact that $>75 \%$ had underlying disease. These findings concur with other studies showing that current vaccination rates among target persons remain low $[26,27]$. The current authors believe that a wider use of the pneumococcal polysaccharide vaccine may help to prevent bacteraemic pneumococcal pneumonia and, conceivably, lowering the rates of early deaths in CAP.

However, the present data suggest a possible relationship between early deaths and discordant therapy in cases of $P$. aeruginosa pneumonia, since five out of six patients with early deaths while receiving discordant therapy had this diagnosis. None of these patients had a previous diagnosis of bronchiectasis, nor had they received corticosteroid therapy; thus, they did not present with the major risk factors for $P$. aeruginosa pneumonia indicated in the current guidelines for the management of CAP [1]. Nevertheless, it should be borne in mind that all these patients had severe pneumonia with high risk of death, in spite of appropriate antibiotic therapy.

The clinical risk factors for early mortality identified by multivariate analysis, such as increased age, altered mental status, multilobar pneumonia and shock, have also been recognised in previous studies as factors associated with overall mortality and with mortality occurring within the initial five days after hospital admission [4-7, 28]. Such factors would be expected to influence early evolution if present at admission. In addition, the present study identified chronic heart disease as a factor associated with late mortality. Apart from age (the major driver in the PSI score) the other factors would also be expected to influence early evolution if present at admission. Additionally, the present study identified discordant therapy as an independent risk factor for early mortality. Nevertheless, this finding is supported almost exclusively by the aforementioned cases of patients with $P$. aeruginosa pneumonia, and the issue requires further study. The question of whether the use of appropriate antibiotics has a clear impact on survival in the first hours after admission remains unanswered, and should be addressed by future research.

In conclusion, current early mortality is relatively low, representing around one third of deaths in patients with community-acquired pneumonia who died during hospital admission. It occurs mainly in elderly patients or patients presenting with altered mental status and septic shock. Pneumococcal bacteraemia and discordant antibiotic therapy, mainly due to lack of coverage against Pseudomonas aeruginosa, are also significant risk factors. The major causes of early death are pneumonia related, such as respiratory failure and shock in the setting of an inadequate host response.

\section{REFERENCES}

1 Mandell L, Wunderink R, Anzueto A, et al. Infectious disease society of America/American Thoracic Society Consensus Guidelines on the management of communityacquired pneumonia in adults. Clin Infect Dis 2007; 44: Suppl. 2, S27-S72.

2 File TM. Community-acquired pneumonia. Lancet 2003; 362: 1991-2001.

3 Kyaw MH, Rose CE Jr, Fry AM, et al. The influence of chronic illnesses on the incidence of invasive pneumococcal disease in adults. J Infect Dis 2005; 192: 377-386.

4 Fine MJ, Auble TE, Yealy DM, et al. A prediction rule to identify low-risk patients with community-acquired pneumonia. N Engl J Med 1997; 336: 243-250.

5 Fine MJ, Smith MA, Carson CA, et al. Prognosis and outcomes of patients with community-acquired pneumonia. A meta-analysis. JAMA 1996; 275: 134-141.

6 Mortensen E, Coley C, Singer D, et al. Causes of death for patients with community-acquired pneumonia: results from the Pneumonia Patient Outcomes Research Team Cohort Study. Arch Intern Med 2002; 162: 1059-1064.

7 Marrie TJ, Wu LL. Factors influencing in-hospital mortality in community-acquired pneumonia. A prospective study 
of patients not initially admitted to the ICU. Chest 2005; 127: 1260-1270.

8 Marrie TJ, Durant H, Yates L. Community-acquired pneumonia requiring hospitalization: 5-years prospective study. Rev Infect Dis 1989; 11: 586-599.

9 Davis RB, Iezzoni LI, Phillips RS, Reiley P, Coffman GA, Safran C. Predicting in-hospital mortality: the importance of functional status information. Med Care 1995; 33: 906-921.

10 Mortensen EM, Restrepo MI, Anzueto A, Pugh J. Antibiotic therapy and 48-hour mortality for patients with pneumonia. Am J Med 2006; 119: 859-864.

11 Rosón B, Carratalà J, Fernández-Sabé N, Tubau F, Manresa F, Gudiol F. Causes and factors associated with early failure in hospitalized patients with communityacquired pneumonia. Arch Intern Med 2004; 164: 502-508.

12 Dowell SF, Peeling RW, Boman J, et al. Standardizing Chlamydia pneumoniae assay: recommendations from the Centers for Disease Control and Prevention (USA) and the laboratory centre for disease control (Canada). Clin Infect Dis 2001; 33: 492-502.

13 National Committee for Clinical Laboratory Standards. Methods for Dilution Antimicrobial Susceptibility Test for Bacteria That Grow Aerobically: Approved Standard. $5^{\text {th }}$ Edition. Document M7-A5; supplemental tables M100-S10. Wayne, National Committee for Clinical Laboratory Standards, 2000.

14 National Committee for Clinical Laboratory Standards. Performance Standards for Antimicrobial Susceptibility Testing: Eleventh Informational Supplement. NCCLS document no. M100-S11. Wayne, National Committee for Clinical Laboratory Standards, 2001.

15 Mandell LA, Marrie TJ, Grossman RF, Chow AW, Hyland RH. Canadian guidelines for the initial management of community-acquired pneumonia: an evidencebased update by the Canadian Infectious Diseases Society and the Canadian Thoracic Society. Clin Infect Dis 2000; 31: 383-421.

16 British Thoracic Society Standards of Care Committee. BTS Guidelines for the Management of Community-Acquired Pneumonia in Adults. Thorax 2001; 56: Suppl. 4, IV1-IV64.

17 Halm EA, Fine MJ, Marrie TJ, et al. Time to clinical stability in patients hospitalized with community-acquired pneumonia: implications for practice guidelines. JAMA 1998; 279: 1452-1457.
18 Rosón B, Carratalà J, Dorca J, Casanova A, Manresa F, Gudiol F. Etiology, reasons for hospitalization, risk classes and outcomes of patients with community-acquired pneumonia hospitalized on the basis of conventional admission criteria. Clin Infect Dis 2001; 33: 158-165.

19 Pallares R, Liñares J, Vadillo $\mathrm{M}$, et al. Resistance to penicillin and cephalosporin and mortality from severe pneumococcal pneumonia in Barcelona, Spain. N Engl J Med. 1995; 333: 474-480.

$20 \mathrm{Yu}$ VL, Chiou CC, Feldman C, et al. An international prospective study of pneumococcal bacteremia: correlation with in vitro resistance, antibiotics administered, and clinical outcome. Clin Infect Dis 2003; 37: 230-237.

21 Austrian R, Gold J. Pneumococcal bacteremia with especial reference to bacteremic pneumococcal pneumonia. Ann Intern Med 1964; 60: 759-770.

22 Confalonieri M, Urbino R, Potena A, et al. Hydrocortisone infusion for severe community-acquired pneumonia: a preliminary randomized study. Am J Respir Crit Care Med 2005; 171: 242-248.

23 Garcia-Vidal C, Calbo E, Pascual V, Ferrer C, Quintana S, Garau J. Effects of systemic steroids in patients with severe community-acquired pneumonia. Eur Respir J 2007; 30: 951-956.

24 Fisman DN, Abrutyn E, Spaude KA, Kim A, Kirchner C, Daley J. Prior pneumococcal vaccination is associated with reduced death, complications, and length of stay among hospitalized adults with community-acquired pneumonia. Clin Infect Dis 2006; 42: 1093-1101.

25 Vila-Córcoles A, Ochoa-Gondar O, Llor C, Hospital I, Rodríguez T, Gómez A. Protective effect of pneumococcal vaccine against death by pneumonia in elderly subjects. Eur Respir J 2005; 26: 1086-1091.

26 Greci LS, Katz DL, Jekel J. Vaccinations in pneumonia: pneumococcal and influenza vaccination patterns among patients hospitalized for pneumonia. Prev Med 2005; 40: 384-388.

27 Musher DM, Alexandraki I, Graviss EA, et al. Bacteremic and non-bacteremic pneumococcal pneumonia. A prospective study. Medicine (Baltimore) 2000; 79: 210-221.

28 Férnandez-Sabé N, Carratalà J, Rosón B, et al. Communityacquired pneumonia in very elderly patients, causative organisms, clinical characteristics, and outcomes. Medicine (Baltimore) 2003; 82: 159-169. 